\title{
Haematological Predictors of Recovery in Dengue Cases
}

SUSHMA NAYAR'1, B SRIRANJANI², PRIYADARSHINI SHANMUGAM³

\section{ABSTRACT}

Introduction: Dengue fever is associated with relative increase in haemoglobin, haematocrit and monocyte counts and reduction in platelet counts, eosinophil percentage and absolute eosinophil count. Recovery is marked by decrease in haemoglobin, haematocrit and monocyte percentage and increase in platelet count, eosinophil percentage and absolute eosinophil count.

Aim: To assess the haematological parameters like haemoglobin, haematocrit, monocyte counts, eosinophil counts and absolute eosinophil counts which may be used as a surrogate marker for recovery.

Materials and Methods: This retrospective study was conducted at Chettinad Hospital and Research Institute for a period of three months. Hundred consecutive cases of dengue fever were included in the study. All haematological parameters including haemoglobin, haematocrit, total WBC count, differential count with neutrophils, lymphocytes, monocytes, eosinophils, basophils, platelet counts and absolute eosinophil counts were noted on hospital admission and on recovery.

Results: The different haematological parameters which change statistically significantly during dengue infection and recovery are the haemoglobin, haematocrit, monocytes, eosinophil, absolute eosinophil count and platelet count ( $p$-value<0.001).

Conclusion: These changes in haemoglobin, haematocrit, monocyte percentage and the platelet count, eosinophil percentages and absolute eosinophil count could be used to monitor recovery in dengue cases.

\section{INTRODUCTION}

Dengue fever cause changes in the peripheral blood counts including relative increase in haemoglobin, haematocrit and monocyte counts and reduction in platelet counts, eosinophil percentage and absolute eosinophil count. Haematologically, recovery is marked by decrease in haemoglobin,haematocrit and monocyte percentage and increase in platelet counts,eosinophil percentage and absolute eosinophil counts. Haematological recovery coincides with clinical recovery in all cases [1,2].

In low resource and rural setting, where the automated haematology analysers and skilled technicians may not be available and hence accurate platelet counts may not be possible, a combination of other haematological parameters like haemoglobin, haematocrit, monocyte counts, eosinophil counts and absolute eosinophil counts may be used as a surrogate marker for recovery. This was a feasibility study to assess the same.

Other studies have not highlighted the rebound eosinophilia that occurs with recovery in dengue. Thus, the aim of the study was to assess the haematological parameters like haemoglobin, haematocrit, monocyte counts, eosinophil counts and absolute eosinophil counts which may be used as a surrogate marker for recovery.

\section{MATERIALS AND METHODS}

This retrospective study was conducted at Chettinad Hospital and Research Institute, Chennai, Tamil Nadu, India, for a period of three months. Hundred consecutive cases of dengue fever were included in the study after obtaining Institutional Ethics Committee approval (245 IHEC/5-17) and informed consent of the patient. Since nine parameters were being evaluated a sample size of 90 100 was taken.

Ratio estimate $=$ Benchmark population ${ }^{*} \times$ Linear estimate/Linear estimate of total population. Ratio estimate $=20^{*} \times 2000 / 10,000$. Ratio estimate $=100$

Inclusion criteria: Confirmed cases of dengue fever having positive antibody to dengue specific IgM with detection of Dengue NS1Ag (the method of detection is through enzyme-linked immunosorbent assay) and IgG IgM Test by ELISA.

Exclusion criteria: Any case of fever with thrombocytopenia negative for dengue tests (Dengue NS1Ag and IgG IgM Test).

All haematological parameters including haemoglobin, haematocrit, total WBC count, differential count with neutrophils, lymphocytes, monocytes, eosinophils, platelet counts and absolute eosinophil counts were noted on hospital admission and on recovery. The counts were done using automated analyser Beckman Coulter LH-780. Recovery was defined as the day platelet counts showed an upward trend and correlated with clinical recovery in all cases. The platelet count was done daily for first few days. In some cases, the platelet count was done three times a day in those with marked thrombocytopenia. The pattern of haematological changes during the recovery phase was studied. The Complete Blood Count findings were confirmed by peripheral smear examination by a pathologist.

\section{STATISTICAL ANALYSIS}

The evaluated parameters were represented as box plots and bar diagrams.The mean and standard deviation are tabulated with p-value obtained using paired t-test (Mann Whitney U-test). The area under the Receiver Operator Characteristic (ROC) curve is used to measure the predicting power of test variables and for determining the efficacy of tests in correctly classifying diseased and non-diseased individuals. In general, the ROC analysis shows the curve fit between the true positive rate (TPR or sensitivity) against the false-positive rate (FPR or 1-specificity). True Positive Rate $=$ probability of positive test result /diseased and False Positive Rate $=$ probability of positive test result /not diseased.

\section{RESULTS}

In this study, paired t-tests showed a statistical decrease in haemoglobin, haematocrit and monocyte and a statistical increase in eosinophil, platelets and absolute eosinophil count with recovery (p-value $<0.001$ ) [Table/Fig-1,2] 


\begin{tabular}{|c|c|c|c|c|}
\hline Variables & $\begin{array}{l}\text { At the time } \\
\text { of admission } \\
\text { (Mean } \pm S D \text { ) }\end{array}$ & $\begin{array}{l}\text { On recovery } \\
\text { (Mean } \pm \text { SD) }\end{array}$ & $\begin{array}{c}\text { Paired } \\
\text { t-test } \\
\text { p-value }\end{array}$ & Remarks/Description \\
\hline $\mathrm{Hb}(\mathrm{gm} / \mathrm{dL})$ & $13.4 \pm 2.2$ & $12.8 \pm 2.0$ & $<0.001$ & $\begin{array}{l}\text { Significantly reduced } \\
\text { on recovery }\end{array}$ \\
\hline HCT (\%) & $40.2 \pm 6.6$ & $38.2 \pm 6.1$ & $<0.001$ & $\begin{array}{l}\text { Significantly reduced } \\
\text { on recovery }\end{array}$ \\
\hline $\begin{array}{l}\text { TC (cells/ } \\
\text { cu mm) }\end{array}$ & $7875 \pm 15153$ & $8736 \pm 8368$ & 0.486 & No significant change \\
\hline $\mathrm{N}(\%)$ & $39.9 \pm 18.7$ & $38.4 \pm 15.7$ & 0.404 & No significant change \\
\hline L (\%) & $42.5 \pm 17.2$ & $44.6 \pm 14.7$ & 0.233 & No significant change \\
\hline M (\%) & $14.1 \pm 8.3$ & $12.1 \pm 4.3$ & 0.016 & $\begin{array}{l}\text { Significantly reduced } \\
\text { on recovery }\end{array}$ \\
\hline$E(\%)$ & $2.7 \pm 3.5$ & $4.4 \pm 3.8$ & $<0.001$ & $\begin{array}{l}\text { Significantly increased } \\
\text { on recovery }\end{array}$ \\
\hline B (\%) & $0.5 \pm 0.7$ & $0.4 \pm 0.5$ & 0.416 & No significant change \\
\hline $\begin{array}{l}\text { PLT (cells/ } \\
\text { cu mm) }\end{array}$ & $70115 \pm 83858$ & $177725 \pm 127913$ & $<0.001$ & $\begin{array}{l}\text { Significantly increased } \\
\text { on recovery }\end{array}$ \\
\hline $\begin{array}{l}\text { AEC (cells/ } \\
\text { cu mm) }\end{array}$ & $134.7 \pm 169.7$ & $338 \pm 305$ & $<0.001$ & $\begin{array}{l}\text { Significantly increased } \\
\text { on recovery }\end{array}$ \\
\hline
\end{tabular}

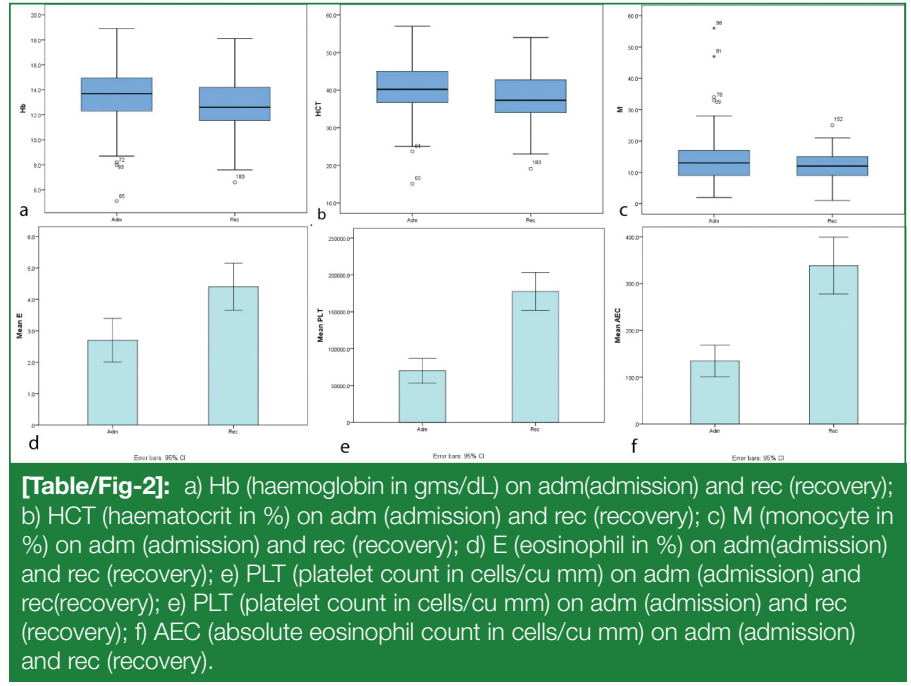

In this study, the area under the curve (AUC) is significantly higher for the parameters haemoglobin, haematocrit, monocyte, eosinophil, platelet count and absolute eosinophil count [Table/Fig-2,3]. The sensitivity and specificity for PLT: $82 \%$ and $88 \%$; for AEC: $82 \%$ and 65\% for $\mathrm{E}: 90 \%$ and $50 \%$ for $\mathrm{Hb} 78 \%$ and $48 \%$; for $\mathrm{HCT}: 77 \%$ and 48\% respectively [Table/Fig-3].

The different haematological parameters which change statistically significantly during dengue infection and recovery are the haemoglobin, haematocrit, monocytes, eosinophil, absolute eosinophil count and platelet count. The total WBC count, neutrophils, lymphocytes and basophils did not change to a statistically significant level in present study. Clinical recovery from dengue infection is marked by a rising platelet count and the haematological parameters paralleled the rising platelet count.

Most cases presented with fever which was high grade and intermittent with chills and rigors for 1-4 days. Few cases had low grade fever. Headache was seen in $28 \%$ of cases. Abdominal pain was seen in $16 \%$ cases, loss of appetite in $10 \%$ of cases, mostly in paediatric cases. Loose stools and rashes in 9\% and 8\% cases respectively. A total of 96 patients had Dengue fever, 4 patients had Dengue Haemorrhagic Fever and none had Dengue Shock Syndrome in this study. Bleeding mostly in the form of haematuria was seen in $4 \%$ cases and rashes were also seen in $4 \%$ cases. Hepatomegaly with right hypochondrial tenderness was seen in $13 \%$ cases. Activated partial thromboplastin time and Prothrombin time was elevated in $8 \%$ cases. An elevation in liver enzymes was seen in $15 \%$ cases. One patient had associated pregnancy with eclampsia,

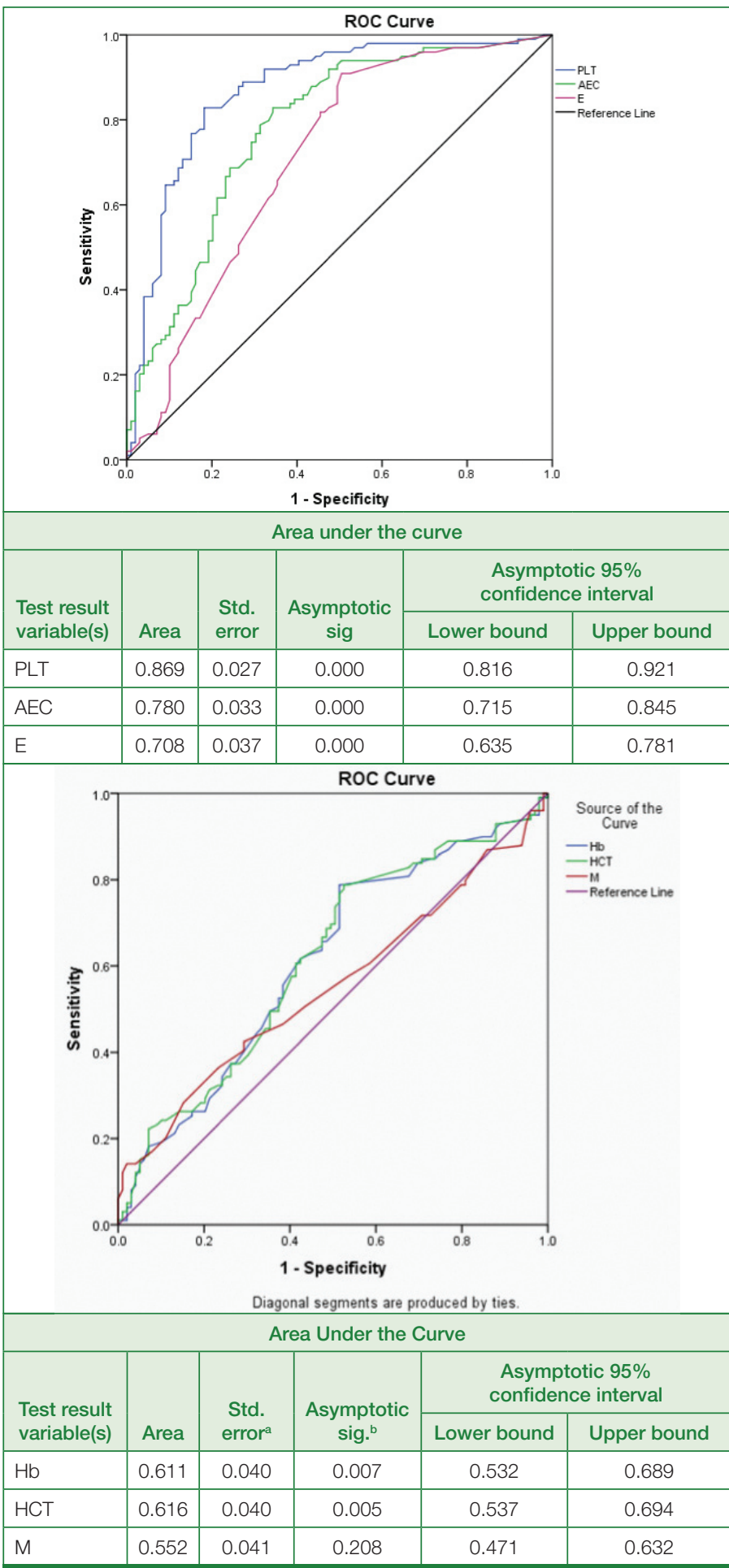

[Table/Fig-3]: The ROC curve analysis for platelet count (PLT), absolute eosinophil count (AEC), and Eosinophil percentage (E); The ROC curve analysis for haemoglobin $(\mathrm{Hb})$, haematocrit $(\mathrm{HCT})$, and Monocyte percentage $(\mathrm{M})$.

who recovered. One patient had associated diabetes mellitus and one paediatric patient with mesenteric adenitis which resolved on treatment. Four patients had platelet transfusion and one patient had fresh frozen plasma transfusion and one patient had packed cell transfusion. All patients recovered and patients with Dengue Haemorrhagic Syndrome had a stormy course and were treated with adequate hydration, platelet transfusions and fresh frozen plasma transfusion. Three Patients with Dengue Haemorrhagic Syndrome recovered while one was discharged against medical advice.

All cases presented with thrombocytopenia which lasted for 3-4 days and for 6-7 days in a few cases. Clinical recovery was marked by a significant rise in platelet count.

\section{DISCUSSION}

The haemoglobin and haematocrit showed an increase in count [1-8]. In a study, there was no significant changes in haematocrit values, 
except for a few patients who had severe bleeding [2]. In another study, the haemoglobin levels remained elevated on discharge in $2.1 \%$ of cases [9]. In another study, the haematocrit increased by $20 \%$ above the levels seen in the convalescent period in $91.5 \%$ patients, in another $8.4 \%$ patients, the haematocrit increased by 10\%-19\% [4]. This is in accordance with our study where the haemoglobin and haematocrit increased during the acute phase. It was more marked in patients with Dengue Haemorrhagic fever. Co-existent anaemia has also been reported. Thrombocytopenia was seen consistently in all studies and an increase heralding recovery [1,2,4-10].

Leucopenia (defined as total WBC count $<4,000$ cells/cu mm)was seen in most studies $[1-3,5,10,11]$. In present study, the total WBC count did not show any statistical difference between the acute phase and convalescent phase of the disease.

Granulocytopenia was seen in one case study [2] and increase in granulocytes during convalescence in another study [8]. Neutropenia was seen $[3,11]$. Lymphocytosis and atypical forms were seen in $93 \%$ patients. Plasmacytoid forms were seen $[3,8]$. An increase in absolute monocyte counts during convalescence were also seen as was noted in this study [11]. An increase in basophils has also been seen in convalescence possibly due to recovery from bone marrow suppression.

Eosinopenia was seen in the acute phase of the disease [2] and a rebound increase in eosinophils was seen with an increase in absolute eosinophil count $[7,8,12]$. A similar finding has been seen with viral infections. In some cases, the eosinophil counts rose more than in the non-infected state [13]. A similar phenomenon has been seen following treatment of filarial infections, where there is rise in eosinophil counts (4 days) after an initial drop in eosinophil counts [14].

\section{LIMITATION}

The reason for the rise of different parameters could not be assessed.

\section{CONCLUSION}

The haemoglobin, haematocrit and monocyte counts rise during the acute phase of the illness and fall during the convalescent phase of the disease. There is a rebound eosinophilia in recovery phase of the disease. As such, these parameters could be used as surrogate markers of recovery in low resource settings and rural areas where an automated haematology analyser may not be available to obtain accurate platelet counts.

\section{REFERENCES}

[1] Fridous J. Dengue Fever (DF) in Pakistan. Asia Pac Fam Med. 2011;10(1):1.

[2] Qiu FX, Gubler DJ, Liu JC, Chen QQ. Dengue in China a clinical review. Bulletin of the World Health Organization. 1993; 71(3/4):349-59.

[3] Thanh HN, Huan-Yao L, Trong LN, Yee-Shin L, Kao-Jean H, Bich LL, et al. Dengue hemorrhagic fever in infants: A study of clinical and cytokine profiles. The Journal of Infectious Diseases. 2004;189(2):221-32.

[4] Eiti FD, Sergio K. Clinical and laboratory characteristics of patients with dengue hemorrhagic fever manifestations and their transfusion profile. Rev Bras Hemato Hemoter. 2014;36(2):115-20.

[5] Shrishu KR, Suchitra R. Clinical features, complications and atypical manifestations of children with severe forms of dengue hemorrhagic fever in South India. Indian J Pediatr. 2006;73(10):889-95.

[6] Kamath S, Jain N, Gupta, Rao BS. Dengue epidemic in Jamshedpur-Tata Main Hospital (TMH) Experience. J Trop Dis. 2015;3:159.

[7] Jameel T, Mehmood K, Mujtaba G, Choudhry N, Afzal N, Paul RF. Changing haematological parameters in dengue viral infections. J Ayub Med Coll Abbottabad. 2012;24(1):03-06.

[8] Beeson, PB, DA Bass. The eosinophile, In Smith LH. (ed.), Major problems in internal medicine, Vol. 14. Philadelphia. WB Saunders Co. 1977;215-34.

[9] Ali N, Usman M, Syed N, Khurshid M. Haemorrhagic manifestations and utility of haematological parameters in dengue fever: A tertiary care centre experience at Karachi. Scand J Infect Dis. 2007;39(11-12):1025-28. Epub 2007 May 30.

[10] Bhaskar ME, Moorthy S, Senthil Kumar N, Arthur P. Dengue haemorrhagic fever among adults - An observational study in Chennai, south India. Indian J Med Res. 2010;132(6):738-40.

[11] Kalayanarooj S, Vaughn DW, Nimmannitya S, Green S, Suntayakorn S, Kunentrasai N, et al. Early clinical and laboratory indicators of acute dengue illness. J Infect Dis. 1997;176(2):313-21.

[12] Pancharoen $C$, Thisyakorn U. Dengue virus infection during infancy. Trans R Soc Trop Med Hyg. 2001;95(3):307-08.

[13] Malathesha MK, Ashwini HN. Hematological manifestations in dengue fever An observational study. Journal of Evolution of Medical and Dental Sciences 2014;3(09:2245-50. DOl: 10.14260/jemds/2014/2133.

[14] Ramya G, Hanna LE, Kumaraswami V, Perumal V, Kavitha V, Vijayasekaran V, et al. Perturbations in eosinophil homeostasis following treatment of lymphatic filariasis infection and immunity. 2000;68(1):93-99.

PARTICULARS OF CONTRIBUTORS:

1. Professor, Department of Pathology, Chettinad Hospital and Research Institute, Kelambakkam, Chennai, Tamil Nadu, India.

2. Student, Chettinad Hospital and Research Institute, Kelambakkam, Chennai, Tamil Nadu, India.

3. Professor and Head, Department of Microbiology, Chettinad Hospital and Research Institute, Kelambakkam, Chennai, Tamil Nadu, India.

NAME, ADDRESS, E-MAIL ID OF THE CORRESPONDING AUTHOR:

Dr. Sushma Nayar

1A, Dolvean Place, 60/3, Taylors Road, Kilpauk, Chennai, Tamil Nadu, India

E-mail: nayarsushma86@gmail.com

Date of Submission: May 24, 2019

Date of Peer Review: Jun 21, 2019

Date of Acceptance: Aug 03, 2019

Date of Publishing: Oct 01, 2019 